\title{
Comparison of three clustering approaches for detecting novel environmental microbial diversity
}

Dominik Forster, Micah Dunthorn, Thorsten Stoeck, Frédéric Mahé

Discovery of novel diversity in high-throughput sequencing studies is an important aspect in environmental microbial ecology. To evaluate the effects that amplicon clustering methods have on the discovery of novel diversity, we clustered an environmental marine high-throughput sequencing dataset of protist reads together with references from the taxonomically curated Protist Ribosomal Reference $\left(\mathrm{PR}^{2}\right)$ database using three de novo approaches: sequence similarity networks, USEARCH, and Swarm. The novel diversity uncovered by each clustering approach differed drastically in the number of operational taxonomic units (OTUs) and in the number of environmental amplicons in these novel diversity OTUs. Global pairwise alignment comparisons revealed that numerous amplicons classified as novel by USEARCH and Swarm were more than $97 \%$ similar to references of $P^{2}$. Using shortest path analyses we found additional novel diversity within OTUs that would have gone unnoticed without further exploiting their underlying network topologies. These results demonstrate that graph theory provides powerful tools for microbial ecology and the analyses of environmental high-throughput sequencing datasets. Furthermore, sequence similarity networks were most accurate in delineating novel diversity from previously discovered diversity. 
4 Comparison of three clustering approaches for

5 detecting novel environmental microbial diversity

6

7

8 Dominik Forster, Micah Dunthorn, Thorsten Stoeck, Frédéric Mahé

9

Department of Ecology, University of Kaiserslautern, Kaiserslautern, Germany

Co-corresponding authors:

16 Dominik Forster, dforster@rhrk.uni-kl.de

17 Frédéric Mahé, mahe@rhrk.uni-kl.de 
19

20

21

\section{ABSTRACT}

Discovery of novel diversity in high-throughput sequencing studies is an important aspect in environmental microbial ecology. To evaluate the effects that amplicon clustering methods have on the discovery of novel diversity, we clustered an environmental marine high-throughput sequencing dataset of protist reads together with references from the taxonomically curated Protist Ribosomal Reference $\left(\mathrm{PR}^{2}\right)$ database using three de novo approaches: sequence similarity networks, USEARCH, and Swarm. The novel diversity uncovered by each clustering approach differed drastically in the number of operational taxonomic units (OTUs) and in the number of environmental amplicons in these novel diversity OTUs. Global pairwise alignment comparisons revealed that numerous amplicons classified as novel by USEARCH and Swarm were more than $97 \%$ similar to references of $\mathrm{PR}^{2}$. Using shortest path analyses we found additional novel diversity within OTUs that would have gone unnoticed without further exploiting their underlying network topologies. These results demonstrate that graph theory provides powerful tools for microbial ecology and the analyses of environmental high-throughput sequencing datasets. Furthermore, sequence similarity networks were most accurate in delineating novel diversity from previously discovered diversity.

Keywords: Environmental diversity inventories; Novel diversity; High-throughput sequencing data; Sequence clustering; Bioinformatics 


\section{INTRODUCTION}

High-throughput sequencing technologies have fundamentally changed our perceptions and concepts of environmental protist diversity (Amaral-Zettler et al., 2009; de Vargas et al., 2015; Logares et al., 2014; Massana et al., 2015; Stoeck et al., 2009). Current high-throughput sequencing surveys analyze protist communities by targeting specific molecular markers, resulting in datasets of many millions of sequencing reads that can address community-comparative, ecosystem-functioning, and novel-diversity questions (Dunthorn et al., 2014b). The detection of novel diversity, in specific, uses a strategy that detects reads distantly related to previously sequenced reference species (e.g. Berney et al., 2013; Dunthorn et al., 2014b; Edgcomb et al., 2011b; Filker et al., 2014; Gimmler and Stoeck 2015; Hartikainen et al., 2014). Following this strategy, groups of sequences that contain both environmental reads and references represent environmental diversity which is covered by taxonomic reference databases, whereas groups of sequences that exclusively contain environmental reads represent novel variants of diversity. Our understanding of protist diversity is far from complete (Pawlowski et al., 2012). While the detection and description of novel protists is a central task, our ability to detect novel diversity in molecular environmental studies is affected by the way reads are clustered into operational taxonomic units (OTUs).

A traditional method of constructing de novo OTUs is by using the popular program USEARCH (Edgar, 2010), though several other similar alternatives exist (e.g. Fu et al., 2012; Ghodsi et al., 2011; Schloss et al., 2009). USEARCH and these other related programs initiate OTUs by selecting an amplicon (i.e., a dereplicated read) to serve as a centroid. Pairwise comparisons score the global sequence similarity of other 
64 amplicons with the centroid. Amplicons with a global sequence similarity to the centroid 65 equal or greater than a given threshold join the OTU. The OTU is then closed, and its

66

67

68 maximal radius (or diameter, depending on the method used) is equal to the global similarity threshold value. There is no consensus on which global similarity threshold value should be used because taxa evolve at different rates (Brown et al., 2015; Caron et al., 2009; Nebel et al., 2011): a 97\% value is commonly used in protist studies (Edgcomb et al., 2011a; Massana et al., 2015), although higher values are also used (Egge et al., 2015).

A second method of constructing de novo OTUs is by using sequence similarity networks (e.g. Forster et al., 2015). Each node in these networks represents one amplicon, and two nodes are connected by an edge only if their amplicons are within a global similarity value that is computed by pairwise alignment scores. Sequence similarity networks seldom result in one single continuous graph, but consist of several subgraphs, so-called connected components which are groups of connected nodes similar to OTUs (Forster et al., 2015). Since additional nodes are added iteratively, the radius of a connected component is not pre-defined, but can be any value, including higher than the global similarity value. As with USEARCH, there is no agreement upon which global similarity threshold should be used. Unlike USEARCH, sequence similarity networks result in OTUs that exhibit an internal network topology which can be further evaluated by methods of graph theory (Bapteste et al., 2012; Bittner et al., 2010; Jachiet et al., 2013; Junker and Schreiber, 2011; Newman, 2010). For instance, assortativity analyses reveal if nodes that share the same trait preferentially connect with each other (Newman, 2003), while centrality analyses give information about the position of a node 
87 in a network and if this node serves as a hub in the network (Newman, 2005). How 88 these and other methods can address ecological questions in high-throughput

89

90

91

92

93

94

sequencing diversity surveys of protists is demonstrated in Forster et al. (2015). To target novel diversity, we focused on shortest path analyses as a straightforward way to measure the distance (expressed as the number of edges that have to be crossed) between two nodes in a network (Alvarez-Ponce et al., 2013; Forster et al., 2015).

A third method to define de novo OTUs is by using the program Swarm (Mahé et al., 2015, 2014). Unlike USEARCH, Swarm relies on an iterative, single-linkage algorithm that uses a small local clustering threshold $d$. This value $d$ is user-defined (1 by default), and corresponds to the maximum number of differences due to substitutions or insertions/deletions between two globally aligned amplicons. Swarm selects the most abundant amplicon available in the amplicon pool to serve as a centroid for a new OTU. All pool amplicons with $d$ or less differences to the centroid are added to the OTU and removed from the pool. Each of those newly added amplicons are compared to the pool amplicons to find those with $d$ or less differences. The process is repeated until no new amplicon can be added to the OTU. To avoid the formation of long chains of amplicons, a classic issue with single linkage clustering, swarm takes into account the abundance of each amplicon (i.e. the number of times it has been observed) and can interrupt the iterative process locally. The combination of these two processes confers to Swarm a high level of stringency and a robustness to changes in initial conditions (e.g., order and abundance of amplicons). Like sequence similarity networks, Swarm results in OTUs whose radii can be any value. Also like sequence similarity networks, the internal 
109 connections between the amplicons in Swarm's OTUs create a network of edges and

110 nodes, which can be evaluated by graph theory.

111 To compare how USEARCH, sequence similarity networks, and Swarm affect our

112 ability to uncover novel diversity in protists, we used amplicon data derived from

113 samples taken in European coastal marine environments. To place the environmental

114 amplicons into a taxonomic context of already known diversity, we relied on the curated

115 Protist Ribosomal Reference database $\left(P R^{2}\right)$ (Guillou et al., 2012). With this

116 combination of environmental and taxonomically-identified amplicons, we asked: i) Do

117 all three clustering approaches predict the same amount of novel diversity? ii) Do

118 network analyses uncover additional novel diversity within OTUs that have underlying

119 network topologies? 
120

121

122

123

124

125

126

127

128

129

130

131

132

133

134

135

136

137

138

139

140

141

142

\section{MATERIAL AND METHODS}

\section{Datasets}

We used already published environmental high-throughput sequencing data from the BioMarKs Consortium (www.biomarks.eu) that sampled microbial eukaryote communities at six near-shore marine sites in Norway, France, Spain, Italy and Bulgaria (e.g. Bittner et al., 2013; Dunthorn et al., 2014a; Logares et al., 2014; Massana et al., 2015). The sample design and sample processing, as well as Roche/454 GS FLX Titanium sequencing of the V4 region of $18 \mathrm{~S}$ rDNA, is detailed in Massana et al. (2015). Quality filtering and chimera check of the raw reads with both UCHIME (Edgar, 2010) and ChimeraSlayer (Haas et al., 2011) is also outlined in Massana et al. (2015). The 1,476,249 cleaned V4 DNA and RNA reads were dereplicated into 312,503 strictly identical amplicons (dataset provided as a supplementary file). The scripts used to perform the analyses presented in this study can be found online in HTML format (Supplementary File 1).

For reference amplicons, we used the $\mathrm{PR}^{2}$ v203 taxonomic reference database (Guillou et al., 2012). From this database we extracted 115,043 taxonomically identified V4 amplicons. We then combined these reference amplicons with the environmental amplicons for all downstream analyses. The clustering approaches would thus produce OTUs containing a blend of environmental and reference amplicons, or OTUs containing only one type of amplicons. Our goal was to identify differences between the three approaches, focusing on OTUs containing exclusively environmental amplicons, likely to represent novel diversity. 
143

144

145

146

147

148

149

150

151

152

153

154

155

156

157

158

159

160

161

162 163

164

165

\section{Clustering}

Three de novo clustering approaches were used to cluster the combined amplicons. First, USEARCH v8.0.1623 (Edgar, 2010), with a 97\% global similarity value using options -cluster smallmem and -sortedby size. This analysis took 57 seconds on a Linux 2.6 operated machine with dual Intel Xeon E5-2670 processors $(2.6 \mathrm{GHz})$ using 16 physical cores and 64 GB RAM.

Second, basic network topology information was gathered by running a global pairwise alignment analysis in VSEARCH v1.1.3 (https://github.com/torognes/vsearch) using options -allpairs_global and -iddef 1. This analysis took about 5 days on the same computer. The resulting matrix contained $682,621,198$ edges with a weight of at least $97 \%$ global sequence similarity. Based on this matrix we created sequence similarity networks in R version 3.2.1 (http://r-project.org) using 'igraph' scripts (Csardi and Nepusz, 2006). To allow these network analyses in 'igraph', we had to switch to a Linux 2.6 operated machine with dual Intel Xeon E5-4650 processors $(2.7 \mathrm{GHz})$ using 32 physical cores and 256 GB RAM. Usage of less memory or more input data forced an untimely abort of analyses. The sequence similarity networks analyses took 3 hours with this setting.

Third, SWARM v2.1.1 (Mahé et al., 2015, 2014), with $-d=1$ and $-f$. This analysis took 33 seconds on the first computer. Singleton and doubleton OTUs (OTUs consisting of one or two amplicons, respectively) were removed from the results of all three clustering approaches for downstream analyses.

Analyses 
For each clustering approach we distinguished if an OTU consisted of: i) both

167 environmental and reference amplicons, ii) exclusively reference amplicons, and iii)

168

169

170

171

172

173

174

175

176

177

178

179

180

181

182

183

184

185

186

187

188

exclusively environmental amplicons. The number of reads in each OTU was also counted.

To compare the novel diversity uncovered by each clustering approach, we analyzed OTUs consisting of exclusively environmental amplicons. For each amplicon in exclusively environmental OTUs, we conducted global pairwise alignments of these amplicons with all $\mathrm{PR}^{2}$ references using VSEARCH (with the options -allpairs_global, iddef 1 and -id 0.70 ), and recorded the highest percentage of similarity to any reference. This revealed how divergent the novel diversity of each clustering approach was with regard to taxonomically identified references. Considering that a $97 \%$ sequence similarity value is used to delineate between different protist species (Edgcomb et al., 2011a; Massana et al., 2015), we expected each novel diversity amplicon in exclusively environmental OTUs to be less than $97 \%$ similar to its closest reference. We also compared if the same environmental amplicons were classified as novel diversity among the different approaches.

To compare within OTUs, shortest path analyses were conducted within each sequence similarity networks and within each Swarm OTU with 'igraph' scripts. The shortest path concept emerges from graph theory and exploits connections between nodes in a network (Newman, 2010). In this particular case we used shortest path analyses to find the minimal number of edges (i.e. connections) that have to be crossed within an OTU to move from each environmental node (i.e. amplicon) to its closest reference node. If an environmental node and a reference node were directly linked (i.e. 
189 direct neighbors separated by exactly one edge), they exhibited a distance of ' 1 ' to each 190 other. As the edges reflected global sequence similarity values of at least $97 \%$ (in 191 sequences similarity networks), or a local basepair difference of '1' (in Swarm), we 192 defined these environmental nodes as the part of diversity that is well represented by 193 the $\mathrm{PR}^{2}$ reference database. Environmental nodes that were not directly linked to 194 reference nodes exhibited a distance of two edges or more, and were thus indirectly 195 linked. Environmental nodes in OTUs, which exclusively consisted of environmental 196 amplicons, exhibited 'infinite' distances to all reference nodes since no shortest path 197 existed. We defined all environmental nodes with distances of two edges or more to 198 reference nodes as novel variants of diversity that are currently not covered by the $\mathrm{PR}^{2}$ 199 database. 


\section{RESULTS AND DISCUSSION}

201

202

203

204

205

206

207

208

209

210

211

212

213

214

215

216

217

218

219

220

221

222

Contrasting OTU results from three approaches

The number of resulting OTUs varied across the three clustering approaches (Table 1). The fewest OTUs in total were produced by sequence similarity networks $(n=$ $8,202)$. Sequence similarity networks also produced the fewest OTUs containing both environmental and reference amplicons $(n=1,619)$, containing exclusively reference amplicons ( $n=3,138)$, and containing exclusively environmental amplicons $(n=3,445)$. This approach was especially effective in linking environmental and reference amplicons: it had the most amplicons in OTUs containing both types ( $n=253,965$ environmental and $n=54,988$ reference). On the other hand, this also led to fewer amplicons in exclusively environmental OTUs $(n=47,116)$, meaning that sequence similarity networks identified the least novel diversity in terms of both amplicons and OTUs.

USEARCH produced more OTUs in total $(n=12,427)$ and more OTUs $(n=5,342)$ that contained exclusively environmental amplicons $(n=71,337)$. The fraction of novel amplicons was therefore increased by one third in USEARCH compared to sequence similarity networks. These differences in OTU numbers may be due in part to how the two methods use their global clustering values: while connected components in sequence similarity networks grow iteratively, OTUs in USEARCH are restricted to a maximum radius (at most $3 \%$ divergence from the centroid). Amplicons whose sequences are less than $97 \%$ similar to the centroid are consequently placed outside of the OTU, although they might be more than $97 \%$ similar to other amplicons inside the OTU. This behavior of USEARCH and other closely-related methods results in an over- 
223 splitting of OTUs (Flynn et al., 2015; Mahé et al., 2014) compared to sequence similarity

224 networks. Additionally, this behavior also causes OTU instability, meaning that a re-

225 clustering with USEARCH may result in slightly different OTU sizes and membership,

226 especially if the input order of the amplicons is shuffled (He et al., 2015; Mahé et al.,

227 2014). Since both factors are especially important for an accurate detection of novel

228 diversity, we argue that the more conservative results of the sequence similarity

229 networks are less prone to contain amplicons and OTUs that are spuriously classified 230 as novel.

231 Although not tested here, previous studies have shown that all-vs.-all pairwise

232 comparison clustering approaches such as sequence similarity networks generally

233 produce more reliable and stable OTUs than heuristic clustering methods such as

234 USEARCH (Schmidt et al., 2015; Sun et al., 2011). This higher reliability and stability of

235 all-vs.-all pairwise comparisons comes at the cost of extensive computational time

236 (Flynn et al., 2015; Sun et al., 2011), which increases with the square to the number of

237 input sequences (Bik et al., 2012). By calculating a pairwise comparison matrix of the

238 currently largest dataset of near-shore marine protists in Europe, we operated close to

239 the limit of dataset size that can be handled in all-vs.-all current approaches.

240 Compared to both approaches relying on global clustering values, Swarm, with

241 its local clustering value, produced the most OTUs in total $(n=13,240)$. The Swarm

242 approach also produced the most OTUs $(n=6,228)$ that contained exclusively

243 environmental amplicons $(n=81,073)$. These higher numbers of OTUs in total and

244 OTUs containing exclusively environmental amplicons may be due to Swarm's high

245 clustering stringency that iteratively links amplicons with a small number of differences 
246 to each other. On the other hand, these high numbers may be due to missing

247 intraspecific sequence variation in the $\mathrm{PR}^{2}$ reference database, which usually contains

248 only one reference per species. In natural communities, intraspecific genetic variation of

249 microbial organisms may be much more diverse than just a few base pair differences,

250 especially in hypervariable gene regions (Brown et al., 2015; Decelle et al., 2014;

251 Dunthorn et al., 2012; Pernice et al., 2013). But in Swarm, an environmental amplicon

252 that differs by more than one base pair to a reference sequence will be placed into a

253 novel OTU, if there are no intermediate amplicons linking them. As long as reference

254 databases are not designed to cover intraspecific sequence variation, it is a more

255 effective strategy to compute Swarm OTUs from datasets consisting entirely of

256 environmental amplicons, and perform a later taxonomical assignment; e.g. as in de

257 Vargas et al. ( 2015), Filker et al. (2014) and Gimmler and Stoeck (2015).

258

259 Is novel diversity really novel?

260 After the identification of novel variants of OTUs and amplicons, the next step in 261 the discovery of novel diversity is normally the design of specific primers and probes for 262 the targeted recovery of organisms from environmental samples (Edgcomb et al., 263 2011b; Gimmler and Stoeck, 2015; Hartikainen et al., 2014; Orsi et al., 2012; 264 Seenivasan et al., 2013). However, this process is time-, cost-, and labor-intensive. An 265 accurate initial classification of novel diversity by clustering approaches is therefore 266 crucial.

There were 29,059 environmental amplicons that were classified as novel by all 268 three clustering approaches (Figure 1). However, the number of environmental 
269 amplicons classified as novel exclusively by one approach differed dramatically: 1,232

270 in sequence similarity networks, 13,777 in USEARCH, and 40,132 in Swarm. Most

271 environmental amplicons which shared less than $97 \%$ sequence similarity with

272 references in $\mathrm{PR}^{2}$ were congruently classified as novel by all three approaches. But

273 both USEARCH and Swarm classified as novel numerous amplicons that were more

274 than $97 \%$ similar to $\mathrm{PR}^{2}$ references (Figure 2, Supplemental Figure S1). Even though

275 clustering in USEARCH was performed at $97 \%$ similarity to delineate novel

276 environmental amplicons from amplicons representing previously described diversity,

277 we found 15,438 amplicons in exclusively environmental OTUs with more than $97 \%$

278 similarity to $\mathrm{PR}^{2}$ references; for Swarm this fraction amounted to 47,007 amplicons. The

279 even larger estimation of novel diversity by Swarm is caused by a combination of the

280 approach's high clustering stringency and missing intraspecific variation in the $\mathrm{PR}^{2}$

281 database. On the other hand, sequence similarity networks classified no environmental

282 amplicon inadvertently as novel, thereby supporting our argument of more accurate

283 novel diversity detection in the latter approach. We conclude that the conservative

284 results of sequence similarity networks most closely match our definition of how we

285 delineated novel diversity from previously described diversity, for a given global 286 clustering threshold value.

287 Beyond that, $97 \%$ of the novel diversity amplicons in sequence similarity 288 networks were identified as novel by at least one of the other two clustering approaches

289 (Figure 1). On the other hand, the 1,232 amplicons exclusively identified as novel by 290 sequence similarity networks clustered into singletons or doubletons in USEARCH and 291 Swarm and were thus excluded from downstream analyses. The novel diversity 
292 uncovered by sequence similarity networks therefore comes closest to a subset of

293 amplicons detected by all three clustering approaches that is truly less than $97 \%$ similar

294 to references in $\mathrm{PR}^{2}$. Furthermore, we strongly advise to perform an additionally

295 taxonomic assignment step in Swarm and USEARCH to validate if potential novel

296 diversity is indeed highly diverse from deposited references. At the same time, though,

297 we are aware that even amplicons which are highly similar to entries in reference

298 databases may represent novel genetic variants. Such hidden diversity is unlikely to be

299 unveiled by approaches solely relying on global similarity values. Instead, more

300 stringent approaches that trace local substitutions or methods which explore internal

301 OTU structure stand a higher chance of revealing novel genetic variants, since they

302 provide a higher resolution of genetic diversity.

303

304

305

306

307

308

309

310

311

312

313

314

Graph theory allows a more detailed evaluation of high-throughput sequencing datasets

Beyond just being able to relay the number of OTUs, sequence similarity networks and Swarm provided additional underlying information for each of their OTUs in the form of network topologies. As pointed out by Forster et al. (2015), these network topologies can reveal additional within-OTU connections among environmental and reference amplicons by using shortest path analyses.

In sequence similarity networks OTUs that contained both types of amplicons, 239,472 of the 253,965 environmental amplicons were directly connected to reference amplicons (Figure 3). The other 14,493 environmental amplicons were indirectly connected to reference amplicons, and represent novel genetic variation on top of the 47,116 amplicons already placed into exclusively environmental OTUs. In Swarm OTUs 
315 that contained both types of amplicons, only 5,757 of the 142,946 environmental

316 amplicons were directly connected to reference amplicons. The 137,189 environmental

317 amplicons with indirect connections also represent novel genetic variation along side of

318 the 81,073 amplicons in exclusively environmental OTUs. This large number of

319 indirectly connected amplicons in Swarm may be an overestimation because current

320 reference databases do not yet cover intraspecific sequence variation (see above).

321 However, our analyses are a first indication that shortest path analyses are a promising

322 way to explore Swarm OTUs. By analyzing paths within an OTU one could, for example,

323 investigate whether amplicons from the same sampling site are more often directly

324 connected to each other than to amplicons from another site. Thus screening for genetic

325 variation related to regional populations or species.

326 Nevertheless, shortest path analyses are just one way to explore genetic

327 variance and novel diversity within OTUs with network topologies. Graph theory can be

328 used to ask numerous questions in microbial ecology (Junker and Schreiber, 2011;

329 Newman, 2010; Proulx et al., 2005). For instance, analyses of assortativity can indicate

330 if environmental sequences affiliated with a certain habitat more preferentially connect

331 with reference sequences than environmental sequences affiliated with another habitat

332 (Forster et al., 2015), thus revealing which habitat's microbial community is less

333 adequately covered by reference databases.

Conclusions

337 Each of the three clustering approaches provided different perspectives on 338 microbial diversity, while also showing individual weaknesses. Our results corroborate 
339 previous observations of inaccuracy in heuristic clustering approaches and highlight 340 how this inaccuracy also affects the detection of novel diversity. Despite their

341 weaknesses, we argue that the combination of high stringency clustering methods and 342 sequence similarity networks, and the implementation of additional tools from graph 343 theory will be beneficial for the evaluation of high-throughput sequencing datasets. Such 344 tools will uncover underlying patterns from microbial high-throughput sequencing data, 345 which hold important information about environmental microbial communities. 


\section{ACKNOWLEDGEMENTS}

We would like to thank the computational resources at the Regional Computing

348 Center at the University of Kaiserslautern, and the BioMarKs consortium for the data

349 analyzed in this study. We thank the editor, Antonio Fernandez-Guerra, and an 350 anonymous reviewer for constructive comments.

351 
352

353

354

355

356

357

358

359

360

361

362

363

364

365

366

367

368

369

370

371

372

373

374

375

376

377

378

\section{REFERENCES}

Alvarez-Ponce, D., Lopez, P., Bapteste, E., Mclnerney, J.O., 2013. Gene similarity networks provide tools for understanding eukaryote origins and evolution. Proc. Natl. Acad. Sci. 110, E1594-E1603.

Amaral-Zettler, L.A., McCliment, E.A., Ducklow, H.W., Huse S.M., 2009. A method for studying protistan diversity using massively parallel sequencing of V9 hypervariable regions of small-subunit ribosomal RNA genes. PLoS One 4, e6372.

Bapteste, E., Lopez, P., Bouchard, F., Baquero, F., Mclnerney, J.O., Burian, R.M., 2012. Evolutionary analyses of non-genealogical bonds produced by introgressive descent. Proc. Natl. Acad. Sci. 109, 18266-18272.

Berney, C., Romac, S., Mahé, F., Santini, S., Siano, R., Bass, D., 2013. Vampires in the oceans: predatory cercozoan amoebae in marine habitats. ISME J. 7, 2387-2399. doi:10.1038/ismej.2013.116

Bik, H.M., Porazinska, D.L., Creer, S., Caporaso, J.G., Knight, R., Thomas, W.K., 2012. Sequencing our way towards understanding global eukaryotic biodiversity. Trends Ecol. Evol. 27, 233-243. doi:10.1016/j.tree.2011.11.010

Bittner, L., Halary, S., Payri, C., Cruaud, C., Reviers, B. de, Lopez, P., Bapteste, E., 2010. Some considerations for analyzing biodiversity using integrative metagenomics and gene networks. Biol. Direct 5, 1-17.

Bittner, L., Gobet, A., Audic, S., Romac, S., Egge, E.S., Santini, S., Ogata, H., Probert, I., Edvardsen, B., de Vargas, C., 2013. Diversity patterns of uncultured Haptophytes unravelled by pyrosequencing in Naples Bay. Mol. Ecol. 22, 87-101. doi:10.1111/mec.12108

Brown, E.A., Chain, F.J.J., Crease, T.J., Maclsaac, H.J., Cristescu, M.E., 2015. Divergence thresholds and divergent biodiversity estimates: can metabarcoding reliably describe zooplankton communities? Ecol. Evol. 5, 2234-2251. doi:10.1002/ece3.1485 
401

402

403

404

405 406 407

Caron, D.A., Countway, P.D., Savai, P., Gast, R.J., Schnetzer, A., Moorthi, S.D., Dennett, M.R., Moran, D.M., Jones, A.C., 2009. Defining DNA-Based Operational Taxonomic Units for Microbial-Eukaryote Ecology. Appl. Environ. Microbiol. 75, 5797-5808. doi:10.1128/AEM.00298-09

Csardi, G., Nepusz, T., 2006. The igraph software package for complex network research. InterJournal Complex Syst. 1695, 1-9.

Decelle, J., Romac, S., Sasaki, E., Not, F., Mahé, F., 2014. Intracellular Diversity of the V4 and V9 Regions of the 18S rRNA in Marine Protists (Radiolarians) Assessed by High-Throughput Sequencing. PLoS One 9, e104297. doi:10.1371/journal.pone.0104297

de Vargas, C., Audic, S., Henry, N., Decelle, J., Mahé, F., Logares, R., Lara, E., Berney, C., Bescot, N.L., Probert, I., Carmichael, M., Poulain, J., Romac, S., Colin, S., Aury, J.-M., Bittner, L., Chaffron, S., Dunthorn, M., Engelen, S., Flegontova, O., Guidi, L., Horák, A., Jaillon, O., Lima-Mendez, G., Lukeš, J., Malviya, S., Morard, R., Mulot, M., Scalco, E., Siano, R., Vincent, F., Zingone, A., Dimier, C., Picheral, M., Searson, S., Kandels-Lewis, S., Coordinators, T.O., Acinas, S.G., Bork, P., Bowler, C., Gorsky, G., Grimsley, N., Hingamp, P., ludicone, D., Not, F., Ogata, H., Pesant, S., Raes, J., Sieracki, M.E., Speich, S., Stemmann, L., Sunagawa, S., Weissenbach, J., Wincker, P., Karsenti, E., Boss, E., Follows, M., Karp-Boss, L., Krzic, U., Reynaud, E.G., Sardet, C., Sullivan, M.B., Velayoudon, D., 2015. Eukaryotic plankton diversity in the sunlit ocean. Science 348, 1261605. doi:10.1126/science.1261605

Dunthorn, M., Klier, J., Bunge, J., Stoeck, T., 2012. Comparing the Hyper-Variable V4 and V9 Regions of the Small Subunit rDNA for Assessment of Ciliate Environmental Diversity. J. Eukaryot. Microbiol. 59, 185-187. doi:10.1111/j.15507408.2011.00602.x

Dunthorn, M., Otto, J., Berger, S.A., Stamatakis, A., Mahé, F., Romac, S., Vargas, C. de, Audic, S., Consortium, B., Stock, A., Kauff, F., Stoeck, T., 2014a. Placing Environmental Next-Generation Sequencing Amplicons from Microbial Eukaryotes 
408

409

410

411

412

413

414

415

416

417

418

419

420

421

422

423

424

425

426

427

428

429

430

431

432

433

434

into a Phylogenetic Context. Mol. Biol. Evol. 31, 993-1009. doi:10.1093/molbev/msu055

Dunthorn, M., Stoeck, T., Clamp, J., Warren, A., Mahé, F., 2014b. Ciliates and the Rare Biosphere: A Review. J. Eukaryot. Microbiol. 61, 404-409. doi:10.1111/jeu.12121

Edgar, R.C., 2010. Search and clustering orders of magnitude faster than BLAST. Bioinformatics 26, 2460-2461. doi:10.1093/bioinformatics/btq461

Edgcomb, V., Orsi, W., Bunge, J., Jeon, S., Christen, R., Leslin, C., Holder, M., Taylor, G.T., Suarez, P., Varela, R., Epstein, S., 2011. Protistan microbial observatory in the Cariaco Basin, Caribbean. I. Pyrosequencing vs Sanger insights into species richness. ISME J. 5, 1344-1356. doi:10.1038/ismej.2011.6

Edgcomb, V.P., Orsi, W., Breiner, H.-W., Stock, A., Filker, S., Yakimov, M.M., Stoeck, T., 2011. Novel active kinetoplastids associated with hypersaline anoxic basins in the Eastern Mediterranean deep-sea. Deep Sea Res. Part Oceanogr. Res. Pap. 58, 1040-1048. doi:10.1016/j.dsr.2011.07.003

Egge, E.S., Johannessen, T.V., Andersen, T., Eikrem, W., Bittner, L., Larsen, A., Sandaa, R.-A., Edvardsen, B., 2015. Seasonal diversity and dynamics of haptophytes in the Skagerrak, Norway, explored by high-throughput sequencing. Mol. Ecol. n/a-n/a. doi:10.1111/mec.13160

Filker, S., Gimmler, A., Dunthorn, M., Mahé, F., Stoeck, T., 2014. Deep sequencing uncovers protistan plankton diversity in the Portuguese Ria Formosa solar saltern ponds. Extremophiles 19, 283-295. doi:10.1007/s00792-014-0713-2

Flynn, J.M., Brown, E.A., Chain, F.J.J., Maclsaac, H.J., Cristescu, M.E., 2015. Toward accurate molecular identification of species in complex environmental samples: testing the performance of sequence filtering and clustering methods. Ecol. Evol. 5, 2252-2266. doi:10.1002/ece3.1497

Forster, D., Bittner, L., Karkar, S., Dunthorn, M., Romac, S., Audic, S., Lopez, P., Stoeck, T., Bapteste, E., 2015. Testing ecological theories with sequence similarity 
networks: marine ciliates exhibit similar geographic dispersal patterns as multicellular organisms. BMC Biol. 13, 16. doi:10.1186/s12915-015-0125-5

Fu, L., Niu, B., Zhu, Z., Wu, S., Li, W., 2012. CD-HIT: accelerated for clustering the next-generation sequencing data. Bioinformatics 28, 3150-3152. doi:10.1093/bioinformatics/bts565

Ghodsi, M., Liu, B., Pop, M., 2011. DNACLUST: accurate and efficient clustering of phylogenetic marker genes. BMC Bioinformatics 12, 271. doi:10.1186/1471-2105$12-271$

Gimmler, A., Stoeck, T., 2015. Mining environmental high-throughput sequence data sets to identify divergent amplicon clusters for phylogenetic reconstruction and morphotype visualization. Environ. Microbiol. Rep. 7, 679-686.

Guillou, L., Bachar, D., Audic, S., Bass, D., Berney, C., Bittner, L., Boutte, C., Burgaud, G., Vargas, C. de, Decelle, J., del Campo, J., Dolan, J.R., Dunthorn, M., Edvardsen, B., Holzmann, M., Kooistra, W.H.C.F., Lara, E., Bescot, N.L., Logares, R., Mahé, F., Massana, R., Montresor, M., Morard, R., Not, F., Pawlowski, J., Probert, I., Sauvadet, A.-L., Siano, R., Stoeck, T., Vaulot, D., Zimmermann, P., Christen, R., 2012. The Protist Ribosomal Reference database (PR2): a catalog of unicellular eukaryote Small Sub-Unit rRNA sequences with curated taxonomy. Nucleic Acids Res. gks1160. doi:10.1093/nar/gks1160

Haas, B.J., Gevers, D., Earl, A.M., Feldgarden, M., Ward, D.V., Giannoukos, G., Ciulla, D., Tabbaa, D., Highlander, S.K., Sodergren, E., Methé, B., DeSantis, T.Z., Consortium, T.H.M., Petrosino, J.F., Knight, R., Birren, B.W., 2011. Chimeric $16 S$ rRNA sequence formation and detection in Sanger and 454-pyrosequenced PCR amplicons. Genome Res. 21, 494-504. doi:10.1101/gr.112730.110

Hartikainen, H., Ashford, O.S., Berney, C., Okamura, B., Feist, S.W., Baker-Austin, C., Stentiford, G.D., Bass, D., 2014. Lineage-specific molecular probing reveals novel diversity and ecological partitioning of haplosporidians. ISME J. 8, 177-186. doi:10.1038/ismej.2013.136 
463 He, Y., Caporaso, J.G., Jiang, X.-T., Sheng, H.-F., Huse, S.M., Rideout, J.R., Edgar,

464

465

466

467

468

469

470

471

472

473

474

475

476

477

478

479

480

481

482

483

484

485

486

487

488

489

490

R.C., Kopylova, E., Walters, W.A., Knight, R., others, 2015. Stability of operational taxonomic units: an important but neglected property for analyzing microbial diversity. Microbiome 3, 20.

Jachiet, P.-A., Pogorelcnik, R., Berry, A., Lopez, P., Bapteste, E., 2013. MosaicFinder: identification of fused gene families in sequence similarity networks. Bioinformatics 29, 837-844.

Junker, B.H., Schreiber, F., 2011. Analysis of Biological Networks. John Wiley \& Sons.

Logares, R., Audic, S., Bass, D., Bittner, L., Boutte, C., Christen, R., Claverie, J.-M., Decelle, J., Dolan, J.R., Dunthorn, M., Edvardsen, B., Gobet, A., Kooistra, W.H.C.F., Mahé, F., Not, F., Ogata, H., Pawlowski, J., Pernice, M.C., Romac, S., Shalchian-Tabrizi, K., Simon, N., Stoeck, T., Santini, S., Siano, R., Wincker, P., Zingone, A., Richards, T.A., de Vargas, C., Massana, R., 2014. Patterns of Rare and Abundant Marine Microbial Eukaryotes. Curr. Biol. 24, 813-821. doi:10.1016/j.cub.2014.02.050

Mahé, F., Rognes, T., Quince, C., de Vargas, C., Dunthorn, M., 2015. Swarm v2: highlyscalable and high-resolution amplicon clustering. PeerJ.

Mahé, F., Rognes, T., Quince, C., de Vargas, C., Dunthorn, M., 2014. Swarm: robust and fast clustering method for amplicon-based studies. PeerJ 2, e593. doi: $10.7717 /$ peerj.593

Massana, R., Gobet, A., Audic, S., Bass, D., Bittner, L., Boutte, C., Chambouvet, A., Christen, R., Claverie, J.-M., Decelle, J., Dolan, J.R., Dunthorn, M., Edvardsen, B., Forn, I., Forster, D., Guillou, L., Jaillon, O., Kooistra, W.H.C.F., Logares, R., Mahé, F., Not, F., Ogata, H., Pawlowski, J., Pernice, M.C., Probert, I., Romac, S., Richards, T., Santini, S., Shalchian-Tabrizi, K., Siano, R., Simon, N., Stoeck, T., Vaulot, D., Zingone, A., Vargas, C., 2015. Marine protist diversity in European coastal waters and sediments as revealed by high-throughput sequencing. Environ. Microbiol. doi:10.1111/1462-2920.12955 
491 Nebel, M., Pfabel, C., Stock, A., Dunthorn, M., Stoeck, T., 2011. Delimiting operational

492

493

494

495

496

497

498

499

500

501

502

503

504

505

506

507

508

509

510

511

512

513

514

515

516

517 taxonomic units for assessing ciliate environmental diversity using small-subunit rRNA gene sequences. Environ. Microbiol. Rep. 3, 154-158. doi:10.1111/j.17582229.2010.00200.x

Newman, M.E.J., 2003. Mixing patterns in networks. Phys. Rev. E 67, 026126.

Newman, M.E.J., 2005. A measure of betweenness centrality based on random walks. Soc. Netw. 27, 39-54.

Newman, M.E.J., 2010. Networks: an introduction. Oxford University Press.

Orsi, W., Edgcomb, V., Faria, J., Foissner, W., Fowle, W.H., Hohmann, T., Suarez, P., Taylor, C., Taylor, G.T., Vd'ačný, P., Epstein, S.S., 2012. Class Cariacotrichea, a novel ciliate taxon from the anoxic Cariaco Basin, Venezuela. Int. J. Syst. Evol. Microbiol. 62, 1425-1433. doi:10.1099/ijs.0.034710-0

Pawlowski, J., Audic, S., Adl, S., Bass, D., Belbahri, L., Berney, C., Bowser, S.S., Cepicka, I., Decelle, J., Dunthorn, M., Fiore-Donno, A.M., Gile, G.H., Holzmann, M., Jahn, R., Jirků, M., Keeling, P.J., Kostka, M., Kudryavtsev, A., Lara, E., Lukeš, J., Mann, D.G., Mitchell, E.A.D., Nitsche, F., Romeralo, M., Saunders, G.W., Simpson, A.G.B., Smirnov, A.V., Spouge, J.L., Stern, R.F., Stoeck, T., Zimmermann, J., Schindel, D., de Vargas, C., 2012. CBOL Protist Working Group: Barcoding Eukaryotic Richness beyond the Animal, Plant, and Fungal Kingdoms. PLoS Biol 10, e1001419. doi:10.1371/journal.pbio.1001419

Pernice, M.C., Logares, R., Guillou, L., Massana, R., 2013. General Patterns of Diversity in Major Marine Microeukaryote Lineages. PLoS One 8, e57170. doi:10.1371/journal.pone.0057170

Proulx, S.R., Promislow, D.E., Phillips, P.C., 2005. Network thinking in ecology and evolution. Trends Ecol. Evol. 20, 345-353.

Schloss, P.D., Westcott, S.L., Ryabin, T., Hall, J.R., Hartmann, M., Hollister, E.B., Lesniewski, R.A., Oakley, B.B., Parks, D.H., Robinson, C.J., Sahl, J.W., Stres, B., 
518

519

520

521

522

523

524

525

526

527

528

529

530

531

532

533

534

535

536

537

Thallinger, G.G., Horn, D.J.V., Weber, C.F., 2009. Introducing mothur: OpenSource, Platform-Independent, Community-Supported Software for Describing and Comparing Microbial Communities. Appl. Environ. Microbiol. 75, 7537-7541. doi:10.1128/AEM.01541-09

Schmidt, T.S.B., Matias Rodrigues, J.F., von Mering, C., 2015. Limits to robustness and reproducibility in the demarcation of operational taxonomic units. Environ. Microbiol. 17, 1689-1706. doi:10.1111/1462-2920.12610

Seenivasan, R., Sausen, N., Medlin, L.K., Melkonian, M., 2013. Picomonas judraskeda Gen. Et Sp. Nov.: The First Identified Member of the Picozoa Phylum Nov., a Widespread Group of Picoeukaryotes, Formerly Known as "Picobiliphytes." PLoS One 8, e59565. doi:10.1371/journal.pone.0059565

Stoeck, T., Behnke, A., Christen, R., Amaral-Zettler, L., Rodriguez-Mora, M.J., Chistoserdov, A., Orsi, W., Edgcomb, V.P., 2009. Massively parallel tag sequencing reveals the complexity of anaerobic marine protistan communities. BMC Biol. 7, 1-20. doi:10.1186/1741-7007-7-72

Sun, Y., Cai, Y., Huse, S.M., Knight, R., Farmerie, W.G., Wang, X., Mai, V., 2011. A large-scale benchmark study of existing algorithms for taxonomy-independent microbial community analysis. Brief. Bioinform. bbr009. doi:10.1093/bib/bbr009 


\section{Tables and Figures}

539

540 Table 1 Sequence clustering results of the three tested approaches. Indicated

541 is the amount of OTUs and the amount (and type) of amplicons within these OTUs for

542 each class of OTUs defined in our analyses.

543

\begin{tabular}{lccc} 
& USEARCH & $\begin{array}{c}\text { Sequence similarity } \\
\text { networks }\end{array}$ & Swarm \\
\hline OTUs & 12427 & 8202 & 13240 \\
\hline $\begin{array}{l}\text { OTUs containing environmental } \\
\text { and reference amplicons }\end{array}$ & 2527 & 1619 & 1993 \\
\hline Environmental amplicons & 223735 & 253965 & 142946 \\
\hline Reference amplicons & 33386 & 54988 & 18774 \\
\hline $\begin{array}{l}\text { OTUs containing exclusively } \\
\text { reference amplicons }\end{array}$ & 4558 & 3138 & 5019 \\
\hline \multicolumn{1}{c}{ Reference amplicons } & 59368 & 46255 & 49147 \\
\hline $\begin{array}{l}\text { OTUs containing exclusively } \\
\text { environmental amplicons }\end{array}$ & 5342 & 3445 & 6228 \\
\hline Environmental amplicons & 71337 & 47116 & 81073 \\
\hline
\end{tabular}

544

545 
546 Figure 1 Venn-Diagram of the number of amplicons in exclusively

547 environmental OTUs. The area of each clustering approach was proportionally

548 adjusted to the amount of amplicons in exclusively environmental OTUs detected in that

549 approach. Overlapping areas reflect amplicons detected in each of the respective

550 approaches. Numbers indicate how many amplicons are represented by each area

551 whereas each area's size is proportional to the number of amplicons included.

552

553

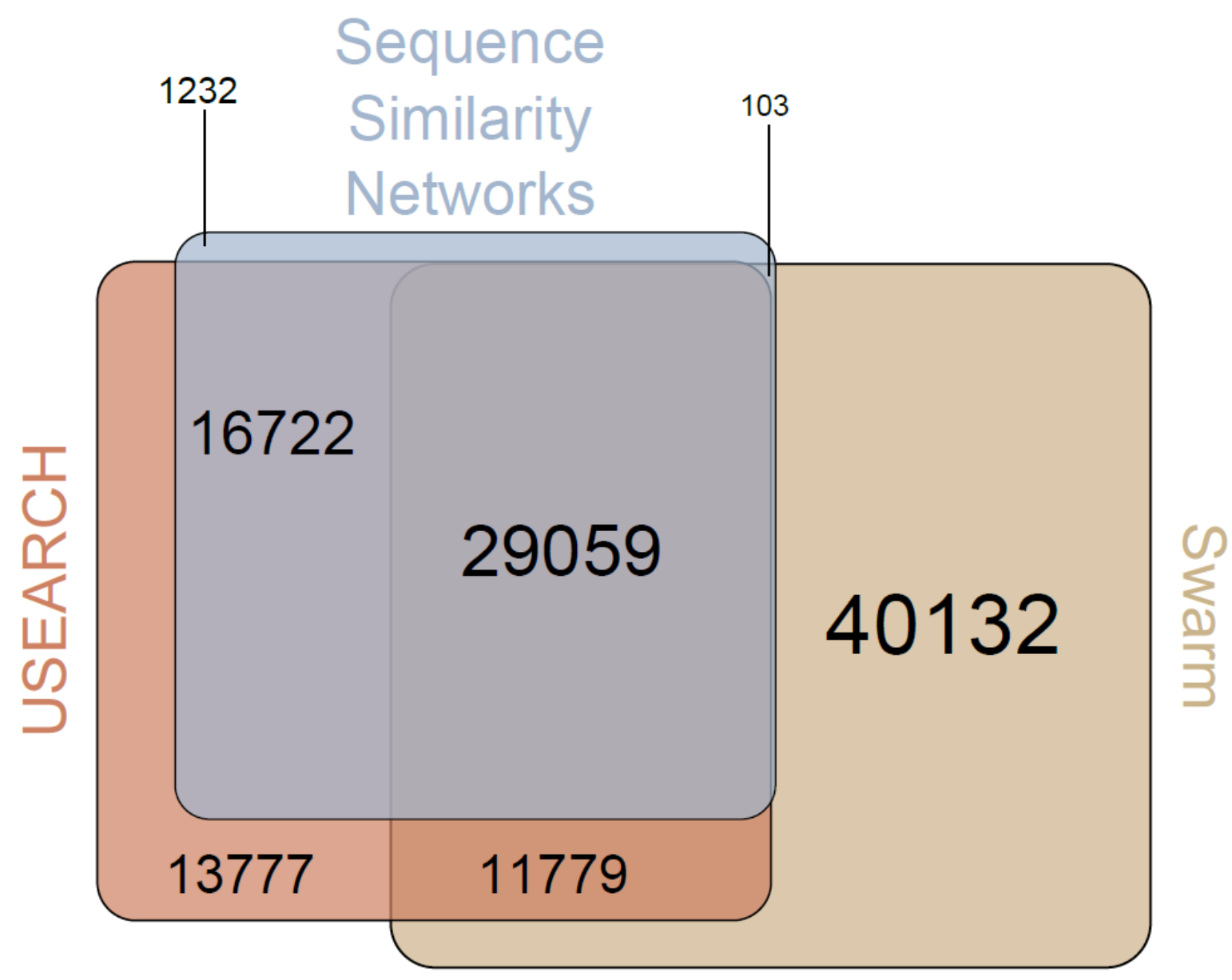


555 Figure 2 Genetic divergence of amplicons in exclusively environmental OTUs

556 to $\mathbf{P R}^{2}$ references by clustering approach. Each point represents one amplicon

557 clustered into an exclusively environmental OTU by the respective clustering approach.

558 Position on the $\mathrm{x}$-axis gives the abundance of each amplicon in the initial dataset before

559 dereplication. The y-axis gives the highest pairwise sequence similarity score of an

560 amplicon to any entry in the $\mathrm{PR}^{2}$ database as calculated by VSEARCH. 

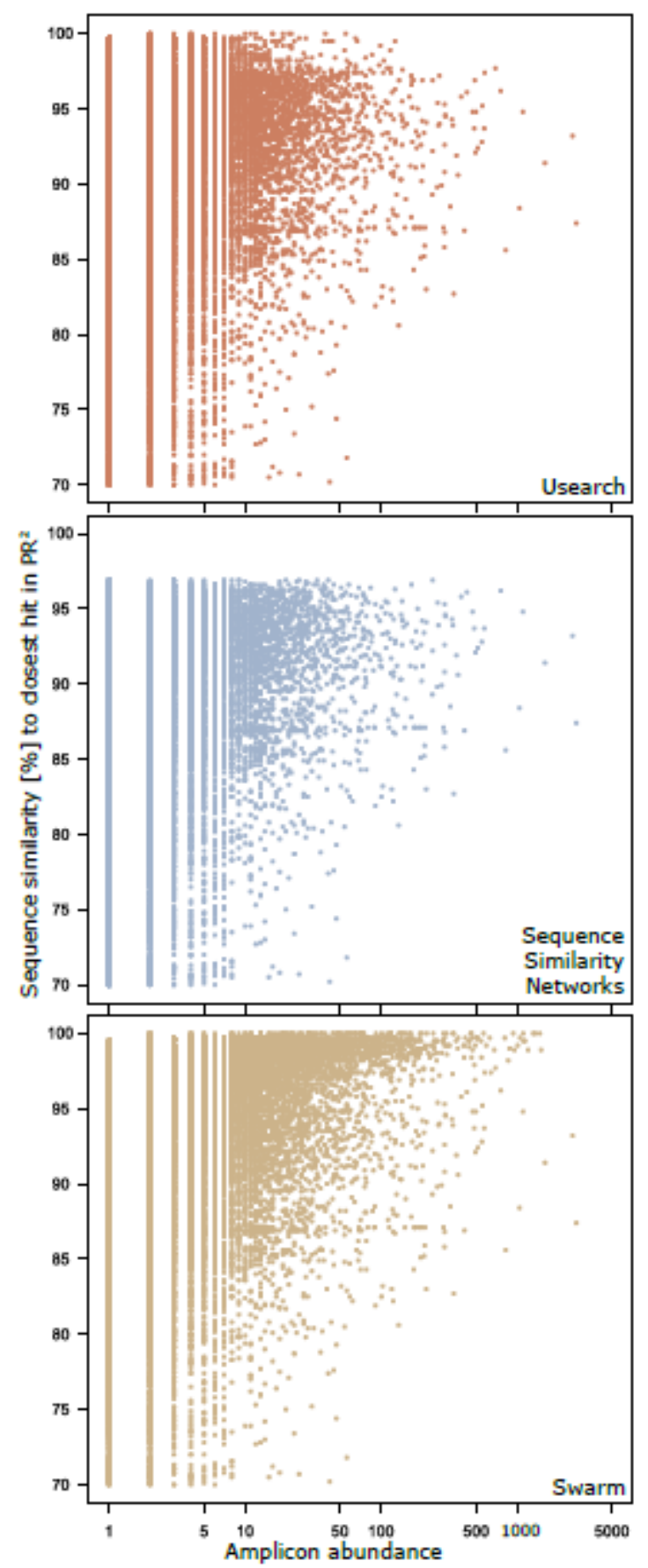

561 
562 Figure 3 Shortest path analyses of CCs and swarms. The plots illustrate how 563 many edges separated each environmental amplicon from its closest reference

564 amplicon in sequence similarity networks and Swarm. A distance of ' 1 ' edge means that 565 the environmental amplicon was directly connected to a reference. 'Infinite' means that 566 the environmental amplicon was placed into an exclusively environmental OTU (see 567 also Table 1) and did not exhibit any connection to a reference amplicon.

568

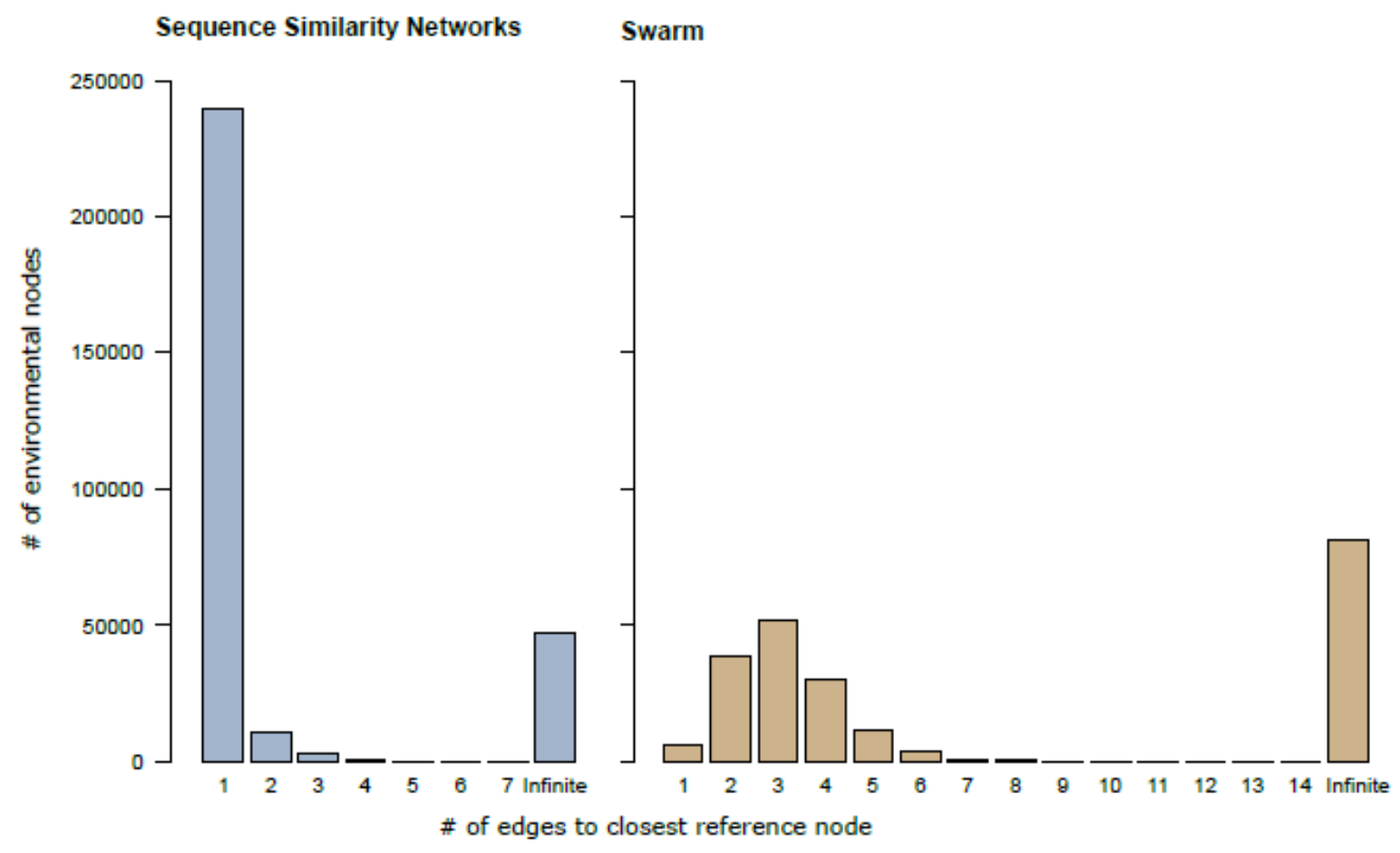

